\title{
Characterisation of a specific class of typical low molecular weight glutenin subunits of durum wheat by a proteomic approach
}

\author{
V. Muccilli ${ }^{a}$, V. Cunsolo ${ }^{a}$, R. Saletti ${ }^{\text {a }}$, S. Foti ${ }^{a}$, B. Margiotta ${ }^{\text {b }}$, F. Scossa ${ }^{c}$, S. Masci ${ }^{c}$, D. Lafiandra ${ }^{\text {c,* }}$ \\ ${ }^{a}$ Department of Chemistry, University of Catania, v.le A. Doria 6, I-95125, Catania,Italy \\ ${ }^{\mathrm{b}}$ Plant Genetics Institute, CNR, Bari, Italy \\ ${ }^{\mathrm{C}}$ Department of Agrobiology and Agrochemistry, University of Tuscia, Viterbo, Italy
}

\section{A R T I C L E I N F O}

\section{Article history:}

Received 5 August 2009

Received in revised form

20 October 2009

Accepted 3 November 2009

\section{Keywords:}

Durum wheat

Low-molecular-weight-glutenin-subunits

Quality

Proteomics

\begin{abstract}
A B S T R A C T
Glutenin polymers are formed by high (HMW-GS) and low molecular weight glutenin subunits (LMWGS). The latter group of subunits has been less characterised compared to the former due to their great number and heterogeneity.

In order to gain more information on the LMW-GS, we have used a durum wheat line carrying a 1BL.1RS translocation, in which the short arm of the chromosome 1B is replaced by the short arm of the chromosome $1 \mathrm{R}$ of rye. This line was obtained using the durum wheat cultivar Cando, in which the translocation is present, crossed and back-crossed four times with the Italian durum wheat cultivar Svevo. Comparative electrophoretic and mass spectrometric analyses carried out on LMW-GS prepared from the durum wheat cultivar Svevo and the line carrying the 1BL.1RS translocation have provided further information on these complex group of proteins. In particular, all the three types of typical LMWGS (LMW-s, LMW-m and LMW-i) were identified in Svevo, whereas the latter group is the only one present in the line with the 1BL.1RS translocation, thus confirming previous findings of the association of the LMW-i type subunits with genes present on chromosome $1 \mathrm{~A}$.
\end{abstract}

(C) 2009 Elsevier Ltd. All rights reserved.

\section{Introduction}

Quality characteristics of durum and bread wheats are strongly influenced by the gluten proteins. They consist of monomeric gliadins (alpha/beta, gamma and omega) characterised by intramolecular disulphide bonds, when present, and polymeric glutenins formed by high (HMW-GS) and low (LMW-GS) molecular weight subunits possessing both intra- and inter-chain bonds. Polymeric glutenins constitute the biggest polymers present in nature, with maximum molecular weights reaching several million Daltons (Wrigley, 1996). Gliadins are encoded at the Gli-1 ( $\omega$ - and $\gamma$ gliadins) and Gli-2 ( $\alpha / \beta$-gliadins) loci, on the short arms of groups 1 and 6 chromosomes, respectively. HMW-GS are encoded at the Glu1 loci, on the long arm of group 1 chromosomes, while LMW-GS are encoded by complex loci on chromosomes 1 and 6, depending on the LMW-GS subgroup (Masci et al., 2002). LMW-GS are, in fact, classified in different groups, according to structural and functional properties. The main subdivision regards the separation between typical LMW-GS (also known as B-subunits), with a peculiar

\footnotetext{
* Corresponding author. Tel.: +390761357243; fax: +390761357238.

E-mail address: lafiandr@unitus.it (D. Lafiandra).
}

structure, and gliadin-like LMW-GS (including C- and D subunits), that are structurally gliadins but functionally glutenins, because they are able to form inter-molecular disulphide bonds by means of unpaired cysteine residues (D’Ovidio and Masci, 2004).

In the case of typical LMW-GS, the sequence of the mature protein can be divided into three parts: a short $\mathrm{N}$-terminal domain, a long central repetitive block and a C-terminal domain where most of the cysteine residues occur. Typical LMW-GS sequences are classified as LMW-s, LMW-m and LMW-i types, on the basis of the first amino acid of the mature polypeptide (serine, methionine or isolecucine, respectively).

On the basis of their ability to form a variable number of intermolecular disulphide bonds, the LMW-GS can be classified as chain extenders or chain terminators. Those subunits forming two interchain disulphide bonds would allow, upon incorporation, the extension of glutenin polymers ("chain extenders", i.e., typical LMW-GS), whereas gliadin-like LMW-GS (chain terminators), once incorporated into a polymer chain, would block their further extension, due to the lack of additional free cysteines for interchain linkages.

B-type subunits include most of the typical LMW-GS sequences, that are the LMW-s, LMW-m and LMW-i types, according to the first amino acid of the mature polypeptide. All typical LMW-GS 